\title{
Accelerated Lane-Changing Trajectory Planning of Automated Vehicles with Vehicle-to-Vehicle Collaboration
}

\author{
Haijian Bai, Jianfeng Shen, Liyang Wei, and Zhongxiang Feng \\ School of Automobile and Traffic Engineering, Hefei University of Technology, Hefei, China \\ Correspondence should be addressed to Jianfeng Shen; 136055068@qq.com
}

Received 13 February 2017; Revised 5 July 2017; Accepted 24 July 2017; Published 28 August 2017

Academic Editor: Xiaobo Qu

Copyright (C) 2017 Haijian Bai et al. This is an open access article distributed under the Creative Commons Attribution License, which permits unrestricted use, distribution, and reproduction in any medium, provided the original work is properly cited.

Considering the complexity of lane changing using automated vehicles and the frequency of turning lanes in city settings, this paper aims to generate an accelerated lane-changing trajectory using vehicle-to-vehicle collaboration (V2VC). Based on the characteristics of accelerated lane changing, we used a polynomial method and cooperative strategies for trajectory planning to establish a lanechanging model under different degrees of collaboration with the following vehicle in the target lane by considering vehicle kinematics and comfort requirements. Furthermore, considering the shortcomings of the traditional elliptical vehicle and round vehicle models, we established a rectangular vehicle model with collision boundary conditions by analysing the relationships between the possible collision points and the outline of the vehicle. Then, we established a simulation model for the accelerated lane-changing process in different environments under different degrees of collaboration. The results show that, by using V2VC, we can achieve safe accelerated lane-changing trajectories and simultaneously satisfy the requirements of vehicle kinematics and comfort control.

\section{Introduction}

In automated environments, the ability to sense surrounding traffic and the ability to control the trajectory of a vehicle in real time are significant factors for the safe and efficient operation of automated vehicles. The existing trajectory planning method is an extension of the path planning method in the field of robotics research. Trajectory planning can result in a smooth and continuous trajectory that satisfies the vehicle kinematics and comfort control requirements. The method proposes finding a global trajectory to connect the origin point to a target point. However, analysing changes in the driving environment requires significant time and is not suitable for handling emergency situations, making it difficult to achieve a global trajectory $[1,2]$. The local trajectory is generated under the guidance of the global trajectory, which is a type of real-time trajectory that is created by sensing the environmental information and can thus be widely used in the trajectory planning of automated vehicles $[3,4]$.

The trajectory of an automated vehicle includes the vehicle following trajectory, the lane-changing trajectory, and the overtaking trajectory. Vehicle following research has rapidly developed, and relevant techniques have been applied in real vehicles. Since overtaking behaviour can be regarded as two occurrences of lane changing, lane-changing trajectory planning has become one of the key components of automated vehicles. Bézier [5, 6], spline [7,8], and polynomial curves $[9,10]$ are currently the must utilized lane-changing trajectory planning methods for automated vehicles. The Bézier curve can continuously generate a lane-changing trajectory using the radius of curvature but is only applicable for static planning because it requires the selection of control points and cannot achieve real-time obstacle avoidance. The spline curve can be used to plan both the arc trajectory and sine trajectory while overcoming the problems associated with the original curve, such as abrupt changes in the curvature and discontinuities; however, spline curves cannot be used to achieve real-time control. The polynomial curve is rapidly calculated, has continuous curvature, and can achieve real-time control. In addition, polynomial curves can be used to obtain the comfort equation using the cubic derivatives.

In automated environments, it is difficult to change lanes because the majority of the vehicles are driving at a high speed, the velocities of the vehicles are stable, and the 


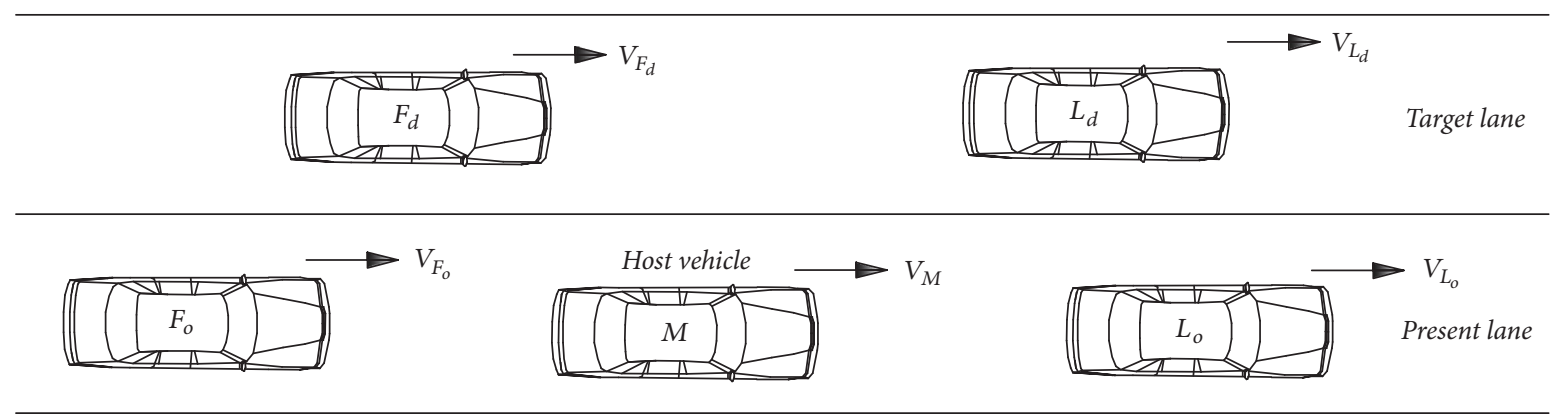

FIgURE 1: Position of the host vehicle relative to the surrounding vehicles.

headway between the vehicles is small. Although overtaking and nonturning lane changes can be reduced or even avoided, mandatory lane changes at road entrances in cities and turning lane changes on urban roads are inevitable. However, the occurrence of vehicle-to-vehicle traffic can require different forms of lane changing because the vehicles can have the same velocity and maintain the same relative position to each other [11].

In this paper, based on automated environments, we generate a strategy using vehicle-to-vehicle collaboration (V2VC) in Section 2. Considering the real-time performance of the polynomial curve and the quantic polynomial obtained from the triple integral of the comfort function, we use the quantic polynomial for trajectory planning in Section 3. Then, using the safety requirements, kinematic requirements, comfort requirements, and cost function of the accelerated lane changing, we determine the constraint function of the optimal trajectory from Section 4 to Section 6. The simulation analysis of a scenario that utilizes the optimal lane-changing trajectory is shown in Section 7.

\section{Strategy of V2VC}

As shown in Figure 1, when the host vehicle $(M)$ changes lanes from the present lane to the target lane, the other vehicles around it include the leading vehicle in the present lane $\left(L_{o}\right)$, the following vehicle in the present lane $\left(F_{o}\right)$, the leading vehicle in the target lane $\left(L_{d}\right)$, and the following vehicle in the target lane $\left(F_{d}\right)$. Among these vehicles, $L_{o}$ and $F_{d}$ have the greatest influence on $M$ when $M$ performs accelerated lane changes. The headway between $M$ and $L_{o}$ determines whether $M$ can accelerate, and the headway between $M$ and $F_{d}$ determines whether $M$ can move to the target lane.

When $M$ intends to change lanes, it will generate safe lane-changing trajectories according to the headway between $M$ and $L_{o}$ and the velocity difference between its current speed and the desired speed. At the same time, a lanechanging signal will be sent to $L_{o}$ and $F_{d}$, which will both provide feedback according to their own driving conditions and the driving state of the nearby vehicles. This V2VC process is shown in Figure 2.

Before changing lanes, the headways between $M$ and $L_{o}$ and between $M$ and $F_{d}$ are $d_{M, L_{o}}$ and $d_{M, F_{d}}$, respectively, and the vehicle speeds are $V_{M}, V_{L_{0}}$ and $V_{F_{d}}$. When $L_{o}$ and $F_{d}$ receive the lane-changing signal, they assess whether or not to accept the lane-changing request according to their current driving state based on the following criteria:

(1) The leading vehicle in the present lane $\left(L_{o}\right)$ : due to the limitations of the vehicles ahead of $L_{o}$, it is difficult for $L_{o}$ to accelerate to provide lane-changing space for $M$, but it can drive at a constant velocity because of the stable traffic flow in the automated environment. After $L_{o}$ receives the lanechanging signal from $M$, it assesses whether it would collide with $M$ while maintaining a constant speed according to the surrounding environment. If it can guarantee that a collision will not occur while driving at a constant speed, it will accept the request; otherwise, it will refuse the request.

(2) The following vehicle in the target lane $\left(F_{d}\right)$ : when $F_{d}$ receives the lane-changing request from $M$, the planned lane-changing trajectories of $M$ are also obtained by $F_{d}$. $F_{d}$ determines whether a collision will occur in the lanechanging process of $M$. If a collision will not occur, $F_{d}$ will accept the lane-changing request and drive at a constant speed; if a collision will occur, $F_{d}$ will consider the relative position of the following vehicle and the requirements of the collaboration degree, decelerate under an acceptable degree of collaboration, and assess whether $M$ will collide with collaborative deceleration. If collision will not occur, $F_{d}$ will accept the lane-changing request and drive at the predetermined deceleration degree of collaboration until $M$ completes the lane change; otherwise, $F_{d}$ will refuse the lanechanging request.

Under the effect of $\mathrm{V} 2 \mathrm{VC}$, when $L_{o}$ and $F_{d}$ receive and accept a lane-changing request, they will change their driving state. To simplify the calculation, we utilize the rectangular coordinate system and regarded the current driving direction of the vehicles as the $X$-axis and the perpendicular direction of travel as the $Y$-axis. Then, we can acquire the driving trajectory of $L_{o}$ and $F_{d}$ under the lane-changing process of $M$.

The driving trajectory of $L_{o}$ is defined as

$$
\begin{aligned}
& X_{L_{o}}(t)=d_{M, L_{o}}+V_{L_{o}} t \\
& Y_{L_{o}}(t)=0
\end{aligned}
$$




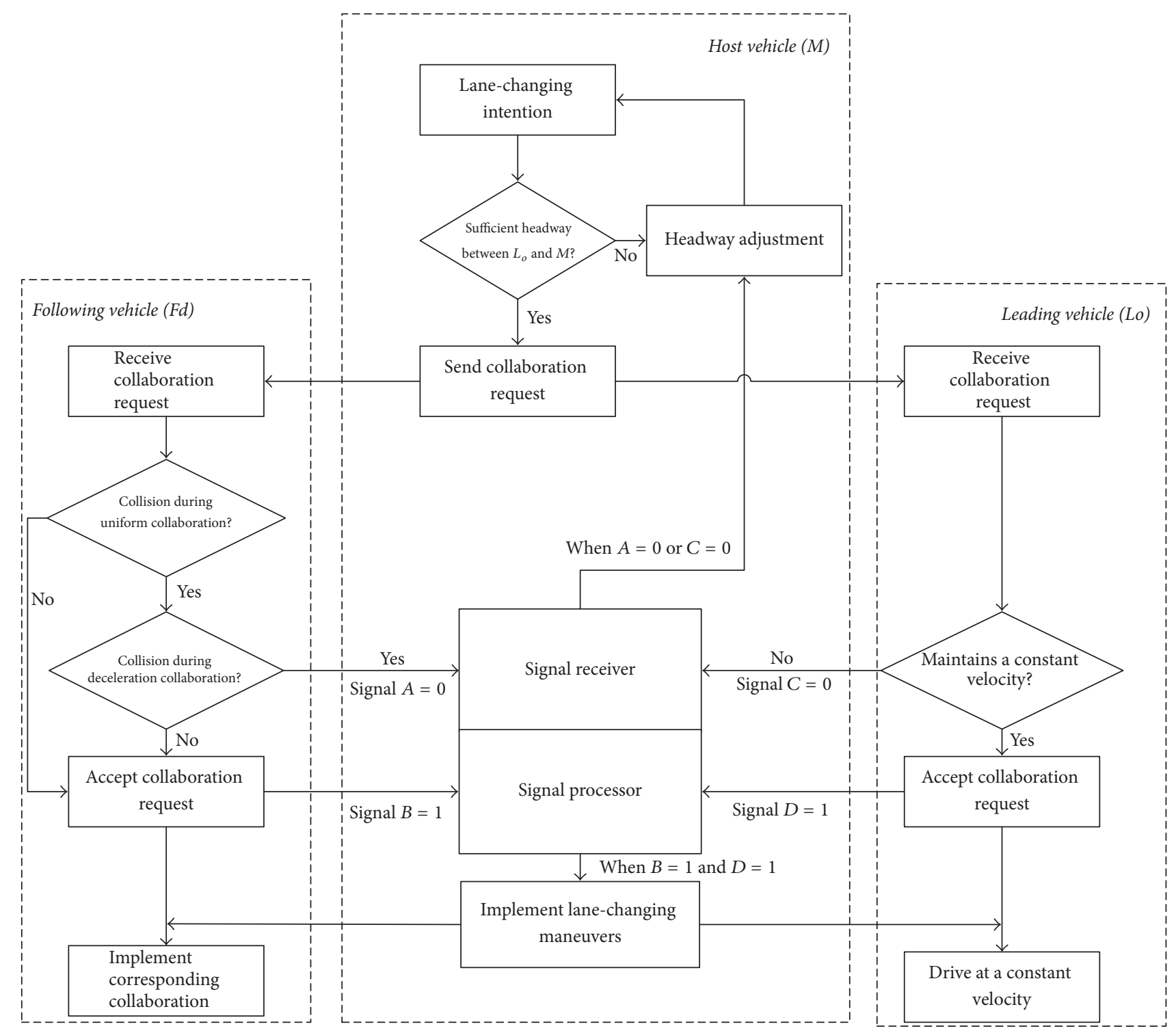

FIGURE 2: Schematic of the V2VC process.

and the driving trajectory of $F_{d}$ is defined as

$$
\begin{aligned}
& X_{F_{d}}(t)=-d_{M, F_{d}}-\frac{1}{2} * a_{F_{d}} t_{F_{d}}{ }^{2}+V_{F_{d}} t_{F_{d}}+V_{F_{d}}^{1}\left(t-t_{F_{d}}\right) \\
& Y_{F_{d}}(t)=W,
\end{aligned}
$$

where $d_{M, L_{o}}$ and $d_{M, F_{d}}$ are the headways between $M$ and $L_{o}$ and between $M$ and $F_{d}$, respectively; $V_{L_{o}}$ and $V_{F_{d}}$ are the initial speeds of $L_{o}$ and $F_{d}$, respectively; $W$ is the horizontal distance from $F_{d}$ to $M$, the value of which is equal to the value of the lane width; $a_{F_{d}}$ is the deceleration of $F_{d}$; and $t_{F_{d}}$ is the time it takes $F_{d}$ to decelerate from its current velocity to an acceptable speed $V_{F_{d}}^{1}$. When $F_{d}$ maintains a constant velocity, $V_{F_{d}}=V_{F_{d}}^{1}$ and $t_{F_{d}}=0$.

\section{Lane-Changing Trajectory Planning of $M$}

The comfort degree is usually described using the jerk of the vehicle during research, and the comfort degree function is often formulated as a quadratic function of time $[3,5]$. The vehicle trajectory is a 5-order polynomial function of time and can be derived from the triple integral of the comfort degree function.

$$
\begin{aligned}
& X_{M}(t)=a_{5} t^{5}+a_{4} t^{4}+a_{3} t^{3}+a_{2} t^{2}+a_{1} t+a_{0} \\
& Y_{M}(t)=b_{5} t^{5}+b_{4} t^{4}+b_{3} t^{3}+b_{2} t^{2}+b_{1} t+b_{0},
\end{aligned}
$$

where $X_{M}(t)$ and $Y_{M}(t)$ represent the longitudinal and lateral coordinates, respectively, which are quantic polynomials with independent variables of time; $a_{i}, i \in\{0,1,2,3,4,5\}$, and 

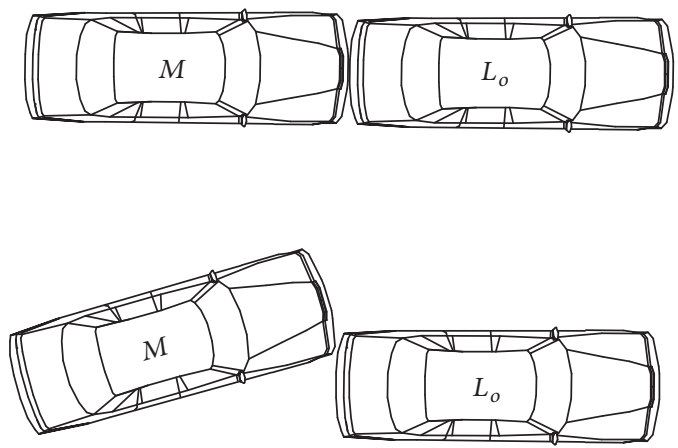

Potential collision types between $M$ and $L_{o}$
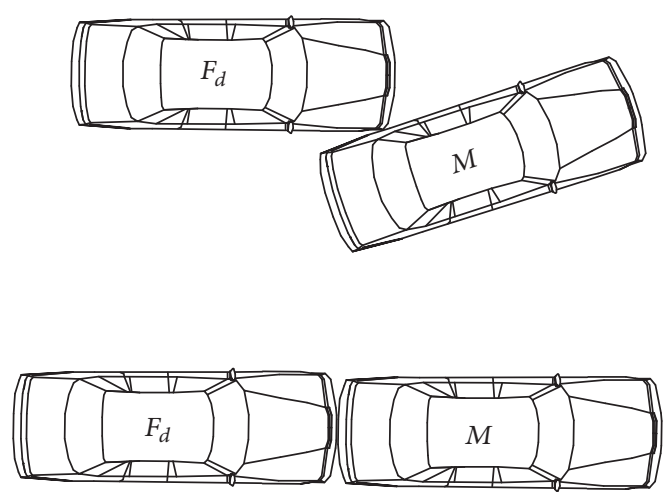

Potential collision types between $M$ and $F_{d}$

FIgURE 3: Collision types between vehicles while changing lanes.

$b_{i}, i \in\{0,1,2,3,4,5\}$, are the parameters of the longitudinal and lateral coordinates of $M$, respectively; and $t$ is the driving time.

Twelve unknown coefficients must be determined in the above functions. Considering the boundary conditions of the initial state and final state of the lane-changing process, we can solve the above equations as follows:

$$
\begin{aligned}
& \text { Initial state: }\left\{\begin{array}{lll}
x(0)=x_{0}, & \dot{x}(0)=v_{x, 0}, & \ddot{x}(0)=a_{x, 0} \\
y(0)=y_{0}, & \dot{y}(0)=v_{y, 0}, & \ddot{y}(0)=a_{y, 0}
\end{array}\right. \\
& \text { Final state: }\left\{\begin{array}{lll}
x\left(t_{c}\right)=x_{c}, & \dot{x}\left(t_{c}\right)=v_{x, c}, & \ddot{x}\left(t_{c}\right)=a_{x, c} \\
y\left(t_{c}\right)=y_{c}, & \dot{y}\left(t_{c}\right)=v_{y, c}, & \ddot{y}\left(t_{c}\right)=a_{y, c},
\end{array}\right.
\end{aligned}
$$

where $x_{0}$ and $y_{0}$ are the current longitudinal and lateral coordinates of $M$, respectively; $v_{x, 0}$ and $v_{y, 0}$ are the current longitudinal and lateral velocities of $M$, respectively; $a_{x, 0}$ and $a_{y, 0}$ are the current longitudinal and lateral accelerations of $M$, respectively; $x_{c}$ and $y_{c}$ are the longitudinal and lateral coordinates of $M$, respectively, when it completes the lanechanging process; $v_{x, c}$ and $v_{y, c}$ are the longitudinal and lateral velocities of $M$, respectively, when it completes the lanechanging process; $a_{x, c}$ and $a_{y, c}$ are the current longitudinal and lateral accelerations of $M$, respectively, when it completes the lane-changing process; and $t_{c}$ is the lane-changing time. Among them, parameters $x_{c}$ and $t_{c}$ are the most difficult to determine, and they are required to solve the quantic polynomial lane-changing trajectory. The solution to this problem is determined in Section 7.

\section{Safety Requirements for Lane Changing}

Previous studies on lane changing have utilized a minimum safe space to ensure that $M$ can take emergency braking precautions to avoid a collision in the event of the sudden deceleration of the leading vehicle. A set of minimum safe distances is used which does not allow for lane changing when the headway between vehicles is too close; these distances are not appropriate in automated environments. The V2VC ensures that the vehicles around $M$ maintain a stable driving state and do not decelerate suddenly when they accept the request of $M$. Therefore, the lane-changing gap can be reduced to the minimum gap, and the lane-changing probability in the automated environment can be increased considerably via V2VC.

4.1. Collision Type during Lane Changing. The safety requirements of the vehicles when changing lanes mainly refer to the collision avoidance requirements of the vehicles. For $M$ to successfully move to the target lane, a collision should be avoided during the lane-changing process if possible. In general, the methods presented in previous studies could not achieve real-time collision avoidance because they only considered the relative positions between the vehicles before lane changing and rarely considered whether collision would occur during the lane-changing process due to changes in the vehicle steering angle.

Vehicle collision accidents can be divided into frontal collisions, rear-end collisions, head-on side collisions, and oblique collisions. During the lane-changing process of $M$, the most likely collisions are rear-end collisions with $L_{o}$ in the original line, oblique collisions with $L_{o}$ during the steering process, oblique collisions during the steering process, and rear-end collisions with $F_{d}$ in the target lane, as shown in Figure 3.

4.2. Selection of the Vehicle Model. During the lane-changing process, the geometric characteristics of the vehicles influence the vehicle collision angle and collision point; thus, an appropriate vehicle model is necessary. Previous studies have regarded the vehicle as a particle to simplify the modelling and calculation processes. Considering the geometric properties of a circle, a few scholars have used a circle around the vehicle to build a vehicle model, as shown in Figure 4(a). However, treating the length of the vehicle as a diameter results in a significant amount of space in the lateral direction which does not contain a vehicle, and the vehicle width can 


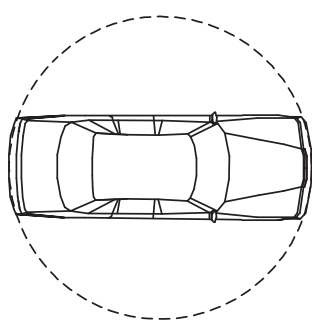

(a) Static circle model

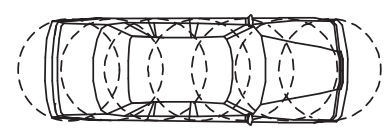

(b) Dynamic circle model

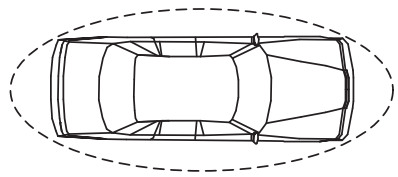

(c) Dynamic ellipse model

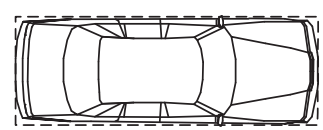

(d) Rectangular vehicle model

FIGURE 4: Schematic diagram of the different vehicle models.

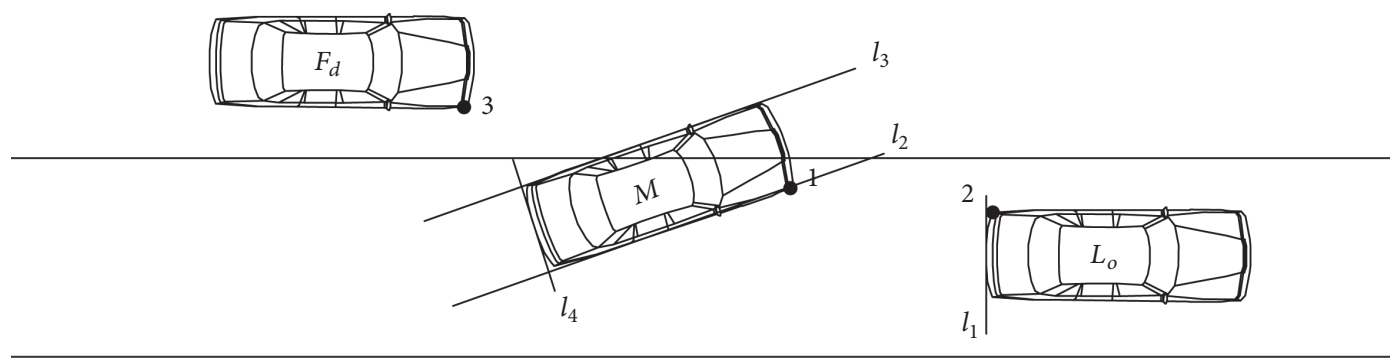

FIGURE 5: Collision relationships between the vehicles during the accelerated lane-changing process.

thus exceed that of a single lane; therefore, this approach is not appropriate for lane-changing maneuvering. A few scholars have used a dynamic circle to cover the entire vehicle, where the width of the vehicle is the diameter of the dynamic circle and the swept area of the dynamic circle is regarded as the vehicle area $[9,12]$, as shown in Figure 4(b). However, this method only considers the physical characteristics of the vehicle and neglects the different collision possibilities in terms of the different driving velocities and the different collision probabilities in the horizontal and vertical directions. Other scholars have used an ellipse to cover the vehicle, as shown in Figure 4(c), where the long half shaft of the ellipse changes with changes in the velocity. Furthermore, determining the appropriate length of the short half shaft is difficult, and steering during the lane-changing process is difficult to achieve $[13,14]$.

Considering the advantages and disadvantages of various vehicle models and combining the requirements to achieve collision avoidance during the lane-changing process, we adopted the rectangular vehicle model, as shown in Figure 4(d). The rectangular vehicle model can accurately simulate the size and contour of the vehicle and is thus convenient for microscopic simulations. However, calculating all distances from the centre point of the vehicle model to the external contour is difficult. A previous analysis of the vehicle lane-changing process indicated that not all of the distances from the centre of the vehicle to the external contour of the vehicle must be calculated. We can assess whether lane changing is feasible as long as we ascertain the possible collision points of the external vehicle contour during the lane-changing process.

4.3. Safety Requirements for Lane Changing with the Rectangular Vehicle Model. During the accelerated lane-changing process, only $M$ is steering; the other vehicles are relatively stable. The potential collision points are shown in Figure 5: collision points 1 and 2 are possible collisions between $M$ and $L_{o}$, and collision point 3 is a possible collision between $M$ and $F_{d}$. To avoid collisions during the lane-changing process, we must assess the relationships between collision point 1 and line 1, collision point 2 and line 2 , and collision point 3 and lines 3 and 4 .

After analysing the above position relationships combined with the driving trajectories of $M, L_{o}$, and $F_{d}$, we can obtain the collision conditions between $L_{o}$ and $M$ :

$$
\begin{aligned}
& X_{L_{o}}(t)-\frac{L_{L_{o}}}{2}-\left(\left(X_{M}(t)+R * \cos (-\beta+\alpha)\right)\right) \leq 0, \\
& \left(Y_{L_{o}}(t)+\frac{w_{L_{o}}}{2}\right)-\left(Y_{M}(t)+R * \sin (-\beta+\alpha)\right)-\tan \alpha \\
& \quad *\left(\left(X_{L_{o}}(t)-\frac{L_{L_{o}}}{2}\right)\right. \\
& \left.\quad-\left(X_{M}(t)+R * \cos (-\beta+\alpha)\right)\right) \geq 0
\end{aligned}
$$


and the collision conditions between $F_{d}$ and $M$ are

$$
\begin{aligned}
& \left(Y_{F_{d}}(t)-\frac{w_{F_{d}}}{2}\right)-\left(Y_{M}(t)+R * \sin (\beta+\alpha)\right)-\tan \alpha \\
& *\left(\left(X_{F_{d}}(t)+\frac{L_{F_{d}}}{2}\right)\right. \\
& \left.\quad-\left(X_{M}(t)+R * \cos (\beta+\alpha)\right)\right) \leq 0, \\
& \left(Y_{F_{d}}(t)-\frac{w_{F_{d}}}{2}\right)-\left(Y_{M}(t)+R * \sin (\pi-\beta+\alpha)\right) \\
& \quad-\tan \left(\alpha+\frac{\pi}{2}\right) *\left(\left(X_{F_{d}}(t)+\frac{L_{F_{d}}}{2}\right)\right. \\
& \left.\quad-\left(X_{M}(t)+R * \cos (\pi-\beta+\alpha)\right)\right) \geq 0,
\end{aligned}
$$

where $L_{L_{o}}$ and $w_{L_{o}}$ are the length and width of $L_{o}$, respectively; $L_{F_{d}}$ and $w_{F_{d}}$ are the length and width of $F_{d}$, respectively; $R=(1 / 2) * \sqrt{L_{M}{ }^{2}+w_{M}^{2}}$ is the radius of rotation along the centroid; $\alpha$ is the steer angle of $M$; and $\beta=$ $\arctan \left(w_{M} / L_{M}\right)$ is the angle of the vertex of the rectangle model with the horizontal direction.

\section{Kinematic and Comfort Requirements of Accelerated Lane Changing}

Considering the accelerated lane-changing characteristics and automated environment described in this paper, we combined previous studies and considered the following kinematic constraints.

(1) The Steering Angle. $0<\Delta \alpha=\arctan \left(\left(\dot{Y}_{M}(t+\Delta T)-\right.\right.$ $\left.\left.\dot{Y}_{M}(t)\right) /\left(\dot{X}_{M}(t+\Delta T)-\dot{X}_{M}(t)\right)\right)<\Delta \alpha_{\max }$, where $\Delta \alpha$ is the change in the vehicle steering angle with an increment of time $(\Delta T), \Delta \alpha_{\max }$ is the change in the maximum vehicle steering angle with $\Delta T$ within the range of maintaining a safe driving performance; $\dot{Y}_{M}(t)$ is the lateral velocity of $M$ at time $t$; and $\dot{X}_{M}(t)$ is the longitudinal velocity of $M$ at time $t$.

(2) Curvature. $0<k=1 / r=\tan \alpha / z_{M}<k_{\max }$, where $k$ is the curvature, $r$ is the turning radius, $z_{M}$ is the wheelbase of the vehicle, and $k_{\max }$ is the maximum curvature of $M$ within the range of maintaining safe driving performance.

(3) Velocity. $0<v_{x, M}(t)<v_{x, \max }$, where $v_{x, M}(t)$ is the longitudinal velocity of $M$ at time $t$ and $v_{x, \max }$ is the maximum longitudinal velocity of the road section.

(4) Position. $0<Y_{M}(t)<W$, where $Y_{M}(t)$ is the lateral displacement of $M$ and $W$ is the lane width.

5.1. Comfort Requirements. Comfort is the subjective feeling of the passengers and is also the key factor for evaluating whether lane changing is reasonable in automated environments. In the model, comfort can be assessed based on the acceleration and jerk in the longitudinal and lateral directions.

$$
\begin{aligned}
& \left|a_{x}\right|<a_{x, \max } \\
& \left|a_{y}\right|<a_{y, \max } \\
& \left|j_{x}\right|<j_{x, \max } \\
& \left|j_{y}\right|<j_{y, \max }
\end{aligned}
$$

where $a_{x}$ and $j_{x}$ are the longitudinal acceleration and jerk, respectively; $a_{y}$ and $j_{y}$ are the lateral acceleration and jerk, respectively; $a_{x, \max }$ and $j_{x, \max }$ are the maximum longitudinal acceleration and jerk, respectively, for the comfort requirements; and $a_{y \text {, max }}$ and $j_{y \text {, max }}$ are the maximum lateral acceleration and jerk, respectively, for the comfort requirements.

\section{Cost Function}

When lane changing occurs, $M$ will have a certain impact on the other vehicles, pedestrians, and traffic flow. To minimize this impact, we established a cost function to evaluate the impact of the lane-changing trajectory. The cost function consists of three components: the comfort level, longitudinal displacement, and lane-changing time.

The comfort level of human beings is an important criterion for evaluating the optimal lane-changing trajectory. The comfort level of a human is defined as follows [15]:

$$
J=\int_{0}^{t_{c}} \dddot{X}_{M}{ }^{2}(t)+\dddot{Y}_{M}{ }^{2}(t) d t
$$

where $\dddot{Y}_{M}(t)$ and $\dddot{X}_{M}(t)$ are the third-order derivatives of the lateral and longitudinal displacement functions of $M$, respectively, indicating the relationship between the jerk and time in the lateral and longitudinal directions, and $j$ is a function of the evaluated comfort level, where a smaller value of $j$ indicates a higher degree of comfort.

The lane-changing longitudinal distance $\left(x_{c}\right)$ is a traffic flow indicator that evaluates the degree of influence of the host vehicle on the surrounding traffic. A smaller value of $x_{c}$ indicates a lesser impact on the traffic flow. The lane-changing time $\left(t_{c}\right)$ is related to the lane-changing efficiency. Considering the influential factors above, we establish the cost function as follows considering the uniform coordination of $F_{d}$ :

$$
\begin{aligned}
C_{\text {con, Cop }}= & w_{1} * \frac{j}{\max (j)}+w_{2} * \frac{x_{c}}{\max \left(x_{c}\right)}+w_{3} \\
& * \frac{t_{c}}{\max \left(t_{c}\right)}
\end{aligned}
$$

where $w_{1}, w_{2}$, and $w_{3}$ are weighted coefficients with a sum of 1 and $\max (j), \max \left(x_{c}\right)$, and $\max \left(t_{c}\right)$ are the comfort function, longitudinal lane-changing distance, and maximum lanechanging time in the feasible trajectory set, respectively.

When $F_{d}$ is used to select the deceleration coordination, its deceleration will have an effect on the surrounding traffic. Considering the negative effects, we establish a cost 


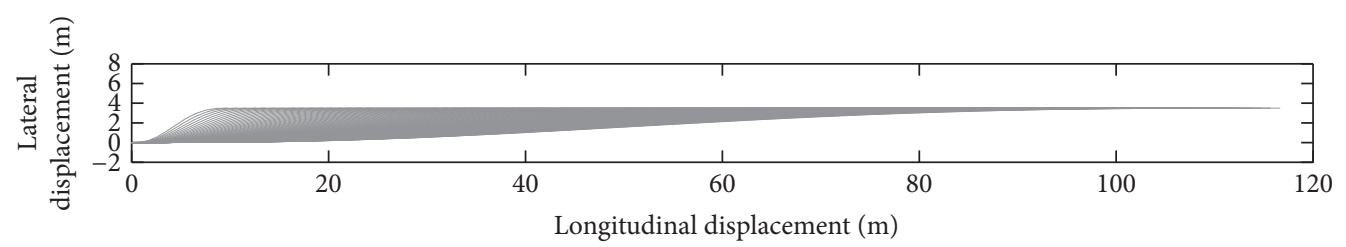

(a) Unconstrained lane-changing trajectory set

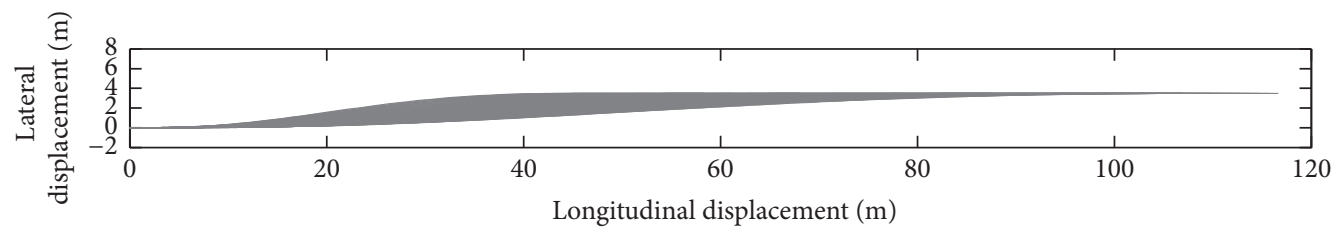

(b) Constrained lane-changing trajectory set



(c) The feasible lane-changing trajectory set

FIGURE 6: Lane-changing trajectory set of $M$ under the uniform coordination of $F_{d}$.

function as follows when $F_{d}$ is used to select the deceleration coordination:

$$
\begin{aligned}
C_{\text {slow }, \text { Cop }}= & \lambda_{1} * \frac{j}{\max (j)}+\lambda_{2} * \frac{x_{c}}{\max \left(x_{c}\right)}+\lambda_{3} \\
& * \frac{t_{c}}{\max \left(t_{c}\right)}+\lambda_{4} * \frac{x_{F_{d}, \text { con }}-x_{F_{d}, \text { slow }}}{x_{F_{d}, \text { con }}},
\end{aligned}
$$

where $\lambda_{1}, \lambda_{2}, \lambda_{3}$, and $\lambda_{4}$ are weighted coefficients with a sum of 1; $x_{F_{d} \text {, slow }}$ is the deceleration collaborative distance of $F_{d}$ during the lane-changing time $\left(t_{c}\right)$; and $x_{F_{d}, \text { con }}$ is the theoretical uniform collaborative distance of $F_{d}$ during $t_{c}$.

\section{Scenario Simulation Analysis}

Based on Matlab simulation software, the uniform coordination, and the deceleration coordination, we studied the differences between the two types of cooperative lanechanging trajectories using a scenario simulation. The main test parameters are as follows: the length and width of the vehicle are $4.5 \mathrm{~m}$ and $1.8 \mathrm{~m}$, respectively; the initial velocity of $M$ is $30 \mathrm{~km} / \mathrm{h}$; the target velocity of $M$ is $40 \mathrm{~km} / \mathrm{h}$ when $M$ completes the lane-changing process; and the current headway between $M$ and $L_{o}$ is $20 \mathrm{~m}$. Considering that the difficulty of solving the quantic polynomial is determining the lanechanging time $\left(t_{c}\right)$ and the longitudinal displacement $\left(x_{c}\right)$, based on the real lane-changing situations, $t_{c}$ is varied from $0 \mathrm{~s}$ to $12 \mathrm{~s}$. Previous studies $[7,16,17]$ have found that when the influence of the comfort requirements is considered, the accelerated lane change will have an approximately constant acceleration process. Thus, the acceleration of $M$ can be obtained, and the longitudinal displacement of the lanechanging process can be determined by determining the lanechanging time $\left(t_{c}\right)$ and speed difference between the initial and final states of $M$.

7.1. Uniform Coordination of $F_{d}$. When space in the target lane is sufficiently large, $F_{d}$ can result in uniform coordination. However, we must avoid a collision between $M$ and $F_{d}$ which could be caused by the sudden acceleration of $F_{d}$. The test parameters are as follows: the velocity of $F_{d}$ is $40 \mathrm{~km} / \mathrm{h}$, the headway between $F_{d}$ and $M$ is $20 \mathrm{~m}$, and the headway between $L_{d}$ and $M$ is $12 \mathrm{~m}$. In this paper, we obtain the unconstrained lane-changing trajectory set based on the quantic polynomial, as shown in Figure 6(a). However, a few trajectories in the unconstrained lane-changing trajectory set do not meet the vehicle kinematic and comfort requirements; thus, we can obtain the constrained trajectory set by deleting these trajectories, as shown in Figure 6(b). At the same time, we acquire the relationship between the velocity and acceleration of the trajectory, as shown in Figure 7. Because of the influence of the comfort level, we can obtain the trajectory that satisfies the requirements when the lane-changing time exceeds $4.8 \mathrm{~s}$ in our simulation. On this basis, we can acquire a practical and feasible lane-changing trajectory set by finding the trajectory that avoids a collision, as shown in Figure 6(c). According to the cost function $\left(C_{\mathrm{con}, \mathrm{Cop}}\right)$ obtained in this simulation, the optimal lane-changing time of $M$ is $7.0 \mathrm{~s}$, and 


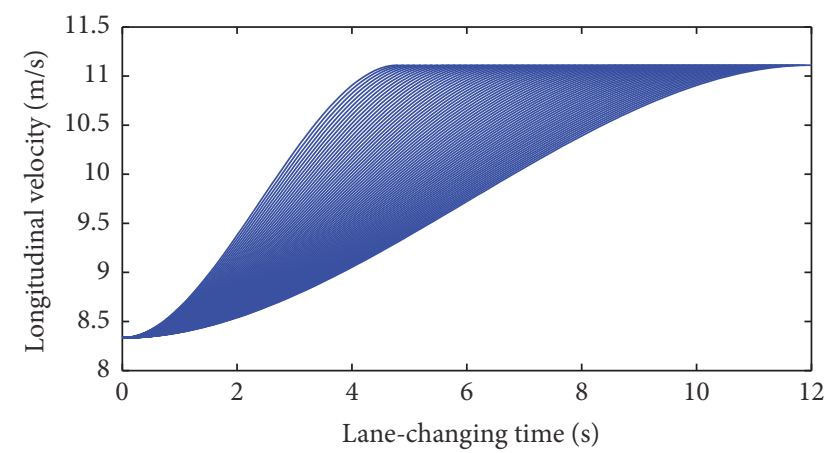

(a) Longitudinal velocity-time diagram $(\mathrm{m} / \mathrm{s})$



(c) Lateral velocity-time diagram $(\mathrm{m} / \mathrm{s})$

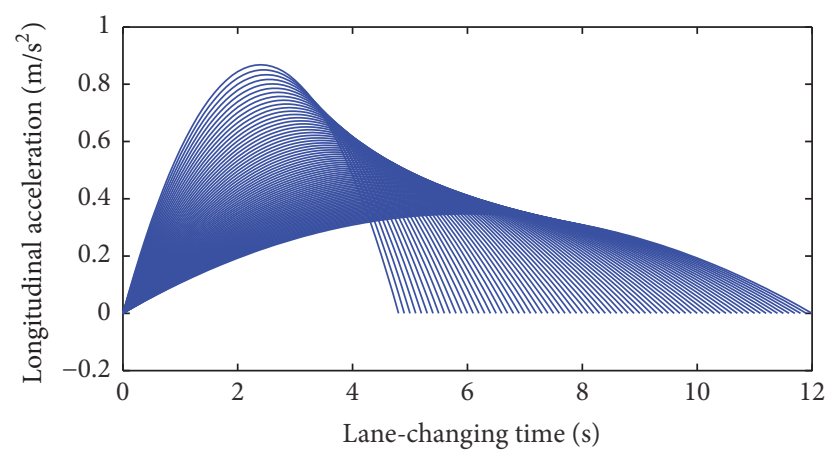

(b) Longitudinal acceleration-time diagram $\left(\mathrm{m}^{2} / \mathrm{s}\right)$



(d) Lateral acceleration-time diagram $\left(\mathrm{m}^{2} / \mathrm{s}\right)$

FIGURE 7: Velocity and acceleration diagrams of the host vehicle $(M)$.

the optimal longitudinal displacement is $68.06 \mathrm{~m}$, which is marked in black in the figure.

After obtaining the optimal trajectory, we conducted a dynamic simulation of the trajectory, as shown in Figure 8 . Through the simulation, the host vehicle can achieve lane changing without collision with a relatively smooth lane-changing trajectory; this behaviour meets the comfort requirements of the passengers in the automated environment.

7.2. Deceleration Coordination of $F_{d}$. When there is insufficient space for the lane-changing process, $F_{d}$ must perform deceleration coordination. The test parameters are as follows: the velocity of $F_{d}$ is $48 \mathrm{~km} / \mathrm{h}$, the cooperative velocity of $F_{d}$ is $40 \mathrm{~km} / \mathrm{h}$, the headway between $F_{d}$ and $M$ is $20 \mathrm{~m}$, and the headway between $L_{d}$ and $M$ is $12 \mathrm{~m}$. The unconstrained trajectory set obtained from the quantic polynomial and the constrained trajectory set that satisfies the kinematic and comfort requirements are the same as those in Figure 6. Because the velocity of $F_{d}$ is too large, $F_{d}$ will collide with $M$ if $M$ maintains a uniform driving velocity. When $F_{d}$ detects this situation, it will decelerate according to the acceptable collaboration degree and follow the predetermined trajectory after accepting the lane-changing request. The feasible lane-changing trajectory set of $M$ is shown in Figure 9. According to the cost function $\left(C_{\text {slow,Cop }}\right)$, we obtain an optimal lane-changing time for $M$ as $6.1 \mathrm{~s}$ in this simulation and an optimal longitudinal displacement of $59.31 \mathrm{~m}$, which is marked in black in the figure. The optimal trajectory simulation of the deceleration coordination of $F_{d}$ is shown in Figure 10.

7.3. Mixture of Uniform and Deceleration Coordination. When the headway between $F_{d}$ and $M$ exceeds a certain amount, the accelerated lane-changing trajectory set of $M$ is divided into two parts: one part is related to the uniform coordination of $F_{d}$ and the other part is related to the deceleration coordination of $F_{d}$. In other words, a few trajectories may not be satisfied under the uniform coordination of $F_{d}$ but will be satisfied under the deceleration coordination of $F_{d}$. Theoretically, the deceleration coordination will increase the lane-changing opportunity of the host vehicle $(M)$, but during the actual process of our simulation, the lane-changing time is generally increased due to the comfort requirements of automated environments; hence, the uniform coordination of $M$ can meet the short lane-changing time. However, when the lane-changing time is excessively long, because the speed of $F_{d}$ is higher than $L_{o}$, the headway between $F_{d}$ and $L_{o}$ is not sufficiently large to satisfy the lane-changing process of $M$ with the uniform coordination of $F_{d}$, as shown in Figure 11. Therefore, the optimal trajectory is located within the part of the lane-changing trajectory set of $M$ which involves the uniform collaboration of $F_{d}$. The test parameters are as follows: the velocity of $F_{d}$ is $48 \mathrm{~km} / \mathrm{h}$, the collaboration degree is $40 \mathrm{~km} / \mathrm{h}$, and the headway between $F_{d}$ and $M$ is $30 \mathrm{~m}$. 


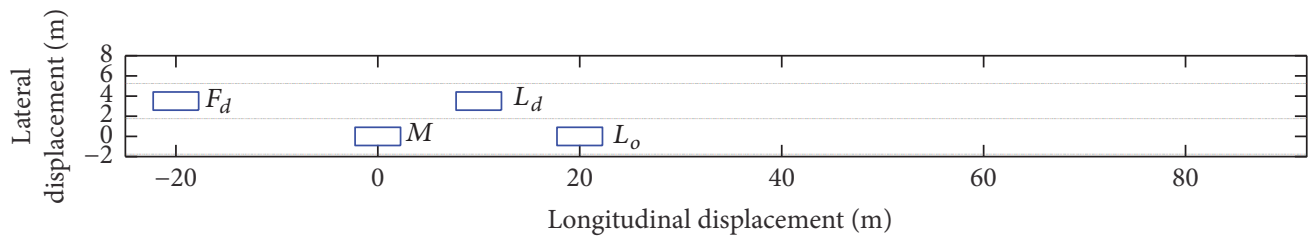

(a) Initial state of the lane-changing process

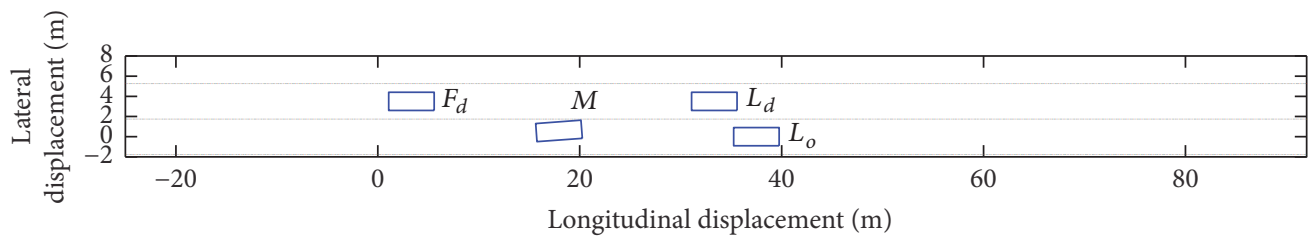

(b) Position relationship between $M$ and $L_{o}$

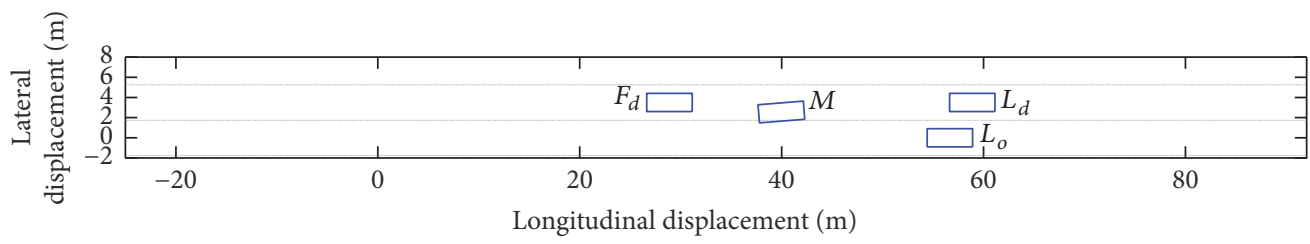

(c) Position relationship between $M$ and $F_{d}$

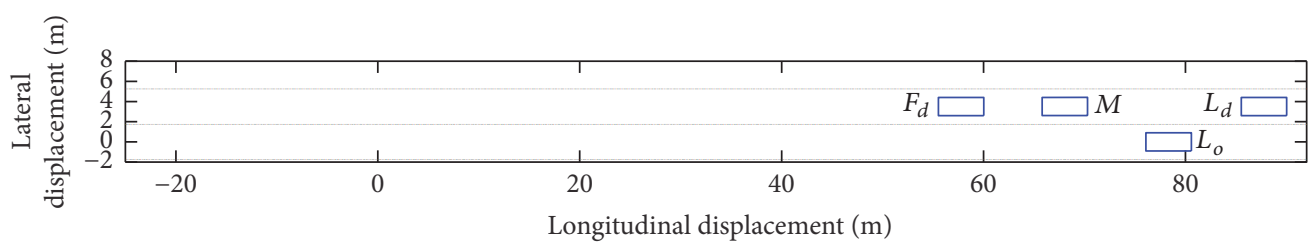

(d) Final state of the lane-changing process

FIGURE 8: Optimal trajectory simulation of $F_{d}$ with uniform coordination.

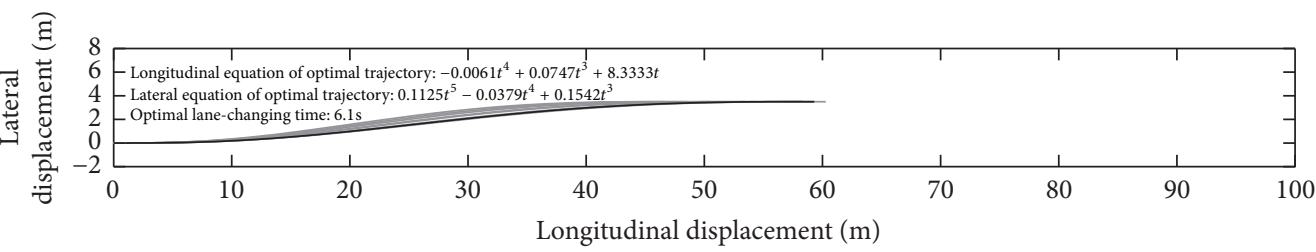

FIGURE 9: Lane-changing trajectory set of $M$ with the deceleration coordination of $F_{d}$.

\section{Conclusion}

In this paper, considering the difficulty of the lane-changing process and the accelerated lane-changing characteristics in an automated environment, we achieved lane changing using V2VC. We studied the accelerated lane-changing process of $M$ with the coordination of $F_{d}$ in an automated environment, and the process was described as a near-uniform acceleration process based on previous studies. Then, we generated an unconstrained lane-changing trajectory set using quantic polynomials, minimized the lane-changing set using actual vehicle kinematic constraints and comfort requirements, and obtained a range of actual trajectories. The results show that we can achieve trajectory planning with the uniform and deceleration collaboration of $F_{d}$. We determined the optimal lane-changing trajectory by calculating the cost function, and the scenario simulations indicated that the generated optimal lane-changing trajectory could achieve safe and comfortable lane changing in an automated environment. 


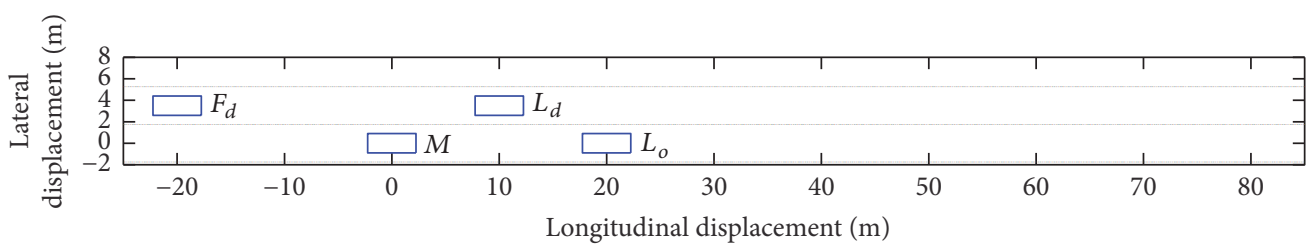

(a) Initial conditions of the lane-changing process

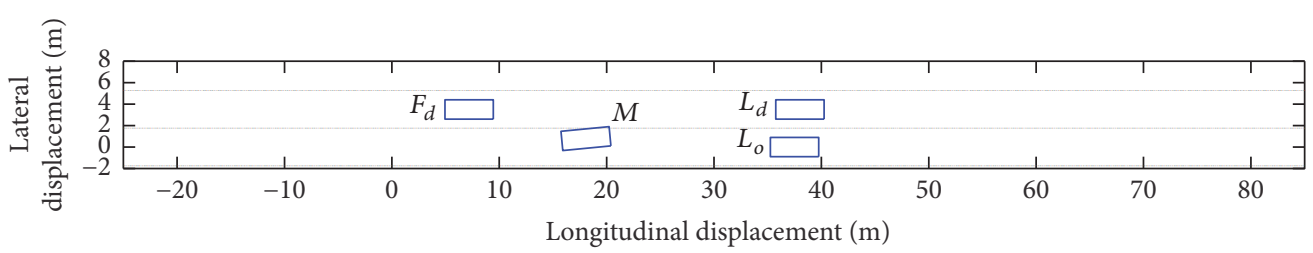

(b) Position relationship between $M$ and $L_{o}$

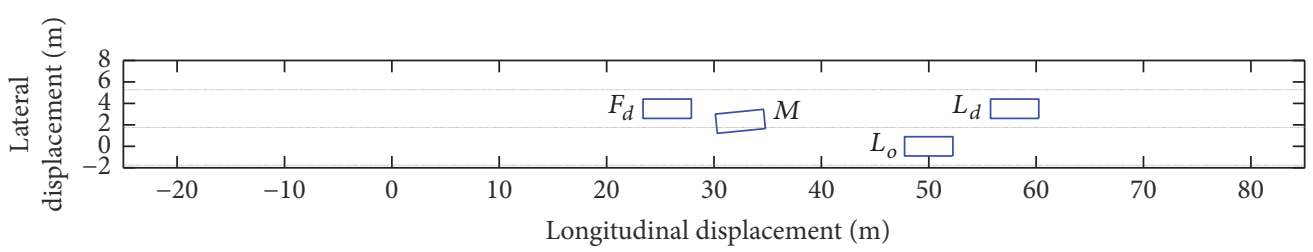

(c) Position relationship between $M$ and $F_{d}$

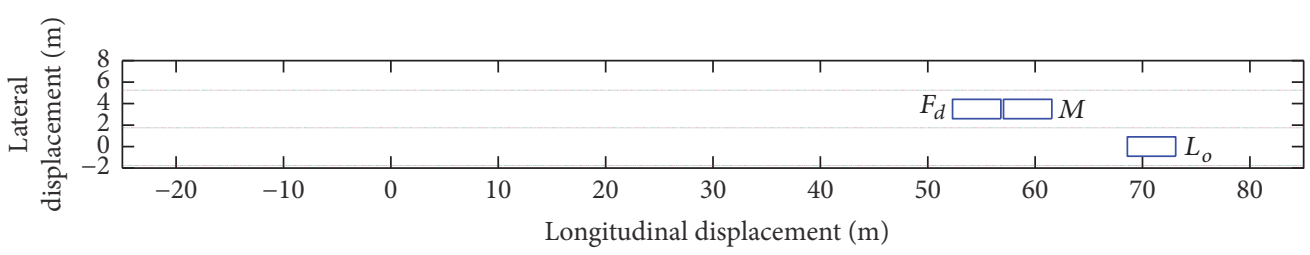

(d) Completion of the lane-changing process

FIGURE 10: Optimal trajectory simulation of the deceleration coordination of $F_{d}$.

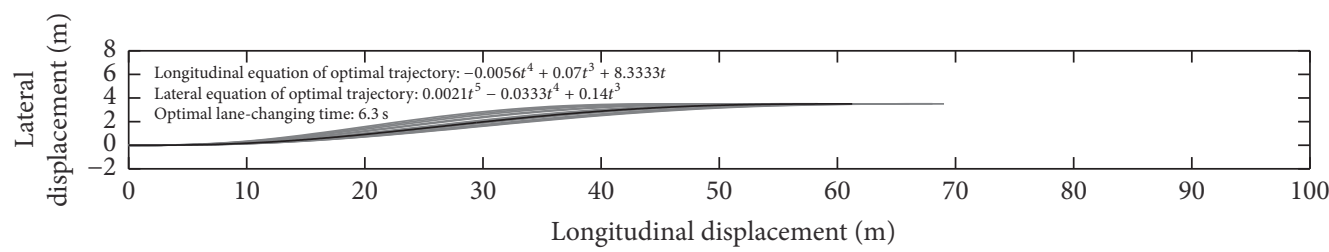

(a) Feasible lane-changing trajectory set of $M$ with the uniform collaboration of $F_{d}$



(b) Feasible lane-changing trajectory set of $M$ with the deceleration collaboration of $F_{d}$

FIGURE 11: The lane-changing trajectory set of $M$ with both the uniform collaboration and deceleration coordination of $F_{d}$.

\section{Conflicts of Interest}

The authors declare that there are no conflicts of interest regarding the publication of this paper.

\section{References}

[1] H.-J. Zhang, J.-W. Gong, Y. Jiang, G.-M. Xiong, and H.-Y. Chen, "An iterative linear quadratic regulator based trajectory tracking 
controller for wheeled mobile robot," Journal of Zhejiang University: Science C, vol. 13, no. 8, pp. 593-600, 2012.

[2] H. Zhang, G. Xiong, B. Su et al., "Anytime path planning in graduated state space," in Proceedings of the 2013 IEEE Intelligent Vehicles Symposium, IEEE IV 2013, pp. 358-362, aus, June 2013.

[3] Y. Luo, Y. Xiang, K. Cao, and K. Li, "A dynamic automated lane change maneuver based on vehicle-to-vehicle communication," Transportation Research Part C: Emerging Technologies, vol. 62, pp. 87-102, 2016.

[4] L. Wei, J. Wang, and D. Jianmin, "Lane changing trajectory planning of intelligent vehicles based on polynomials," Jisuanji Gongcheng yu Yingyong(Computer Engineering and Applications), vol. 48, no. 3, pp. 242-245, 2012.

[5] J.-W. Choi, R. Curry, and G. Elkaim, "Path planning based on bézier curve for autonomous ground vehicles," in Proceedings of the Advances in Electrical and Electronics Engineering IAENG Special Edition of the World Congress on Engineering and Computer Science 2008, WCECS 2008, pp. 158-166, usa, October 2008.

[6] C. Chen, Y. He, C. Bu, J. Han, and X. Zhang, "Quartic Bézier curve based trajectory generation for autonomous vehicles with curvature and velocity constraints," in Proceedings of the 2014 IEEE International Conference on Robotics and Automation, ICRA 2014, pp. 6108-6113, chn, June 2014.

[7] L. Wei, G. Dezhi, and D. Jianmin, "Research on Lane changing Model for Intelligent Vehicles," Journal of Highway and Transportation Research and Development, vol. 27, no. 2, pp. 119-123, 2010.

[8] J. Connors and G. Elkaim, "Analysis of a spline based, obstacle avoiding path planning algorithm," in Proceedings of the 2007 IEEE 65th Vehicular Technology Conference - VTC2007-Spring, pp. 2565-2569, irl, April 2007.

[9] F. You, R. Zhang, G. Lie, H. Wang, H. Wen, and J. Xu, "Trajectory planning and tracking control for autonomous lane change maneuver based on the cooperative vehicle infrastructure system," Expert Systems with Applications, vol. 42, no. 14, pp. 5932-5946, 2015.

[10] M. A. Chuan, Research on Trajectory Planning and Control Algorithm of intelligent Vehicle, Shandong University of Technology, 2013.

[11] M. Zhou, X. Qu, and S. Jin, "On the impact of cooperative autonomous vehicles in improving freeway merging: a modified intelligent driver model-based approach," IEEE Transactions on Intelligent Transportation Systems, vol. PP, no. 99, pp. 1-7, 2016.

[12] Y. Feng, Study on autonomous lane changing and autonomous overtaking control method of intelligent vehicle, College of Traffic, Jilin University, 2005.

[13] L.-h. Xu, S.-g. Hu, and Q. Luo, "Lane-Changing Model Based on Different Types of Drivers," Journal of South China University of Technology (Natural Science Edition), vol. 8, p. 018, 2014.

[14] W. Chonglun, L. Zhenlong, C. Yangzhou, and D. Guiping, "Research on Lane-changing Models Considering Restricted Space [J]," Journal of Highway and Transportation Research and Development, vol. 1, p. 023, 2012.

[15] M. Werling, J. Ziegler, S. Kammel, and S. Thrun, "Optimal trajectory generation for dynamic street scenarios in a frenét frame," in Proceedings of the 2010 IEEE International Conference on Robotics and Automation, ICRA 2010, pp. 987-993, usa, May 2010.

[16] R. Dian-Bo, Z. Jing-Ming, and C. Wang, "Virtual trajectory model for lane changing of a vehicle on curved road with variable curvature," pp. 78902-078902, 2014.
[17] K. Erkorkmaz and Y. Altintas, "High speed CNC system design. Part I. Jerk limited trajectory generation and quintic spline interpolation," International Journal of Machine Tools and Manufacture, vol. 41, no. 9, pp. 1323-1345, 2001. 


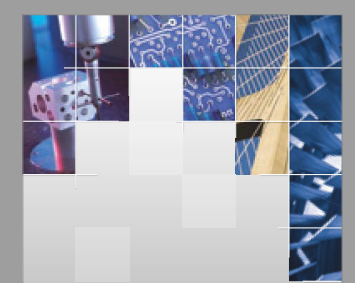

\section{Enfincering}
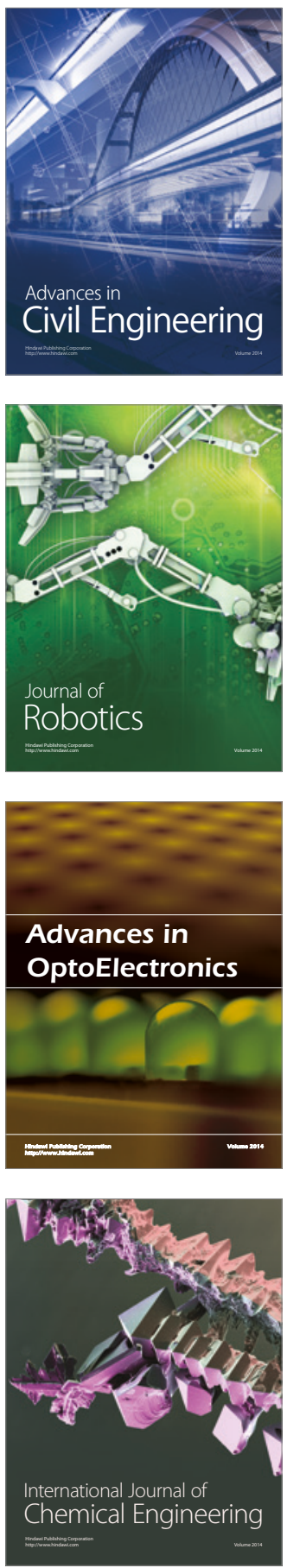

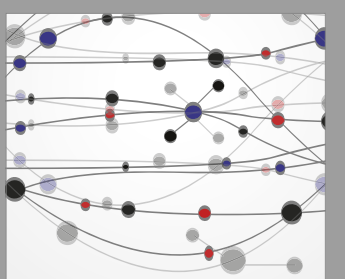

The Scientific World Journal

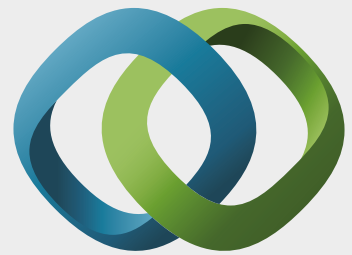

\section{Hindawi}

Submit your manuscripts at

https://www.hindawi.com
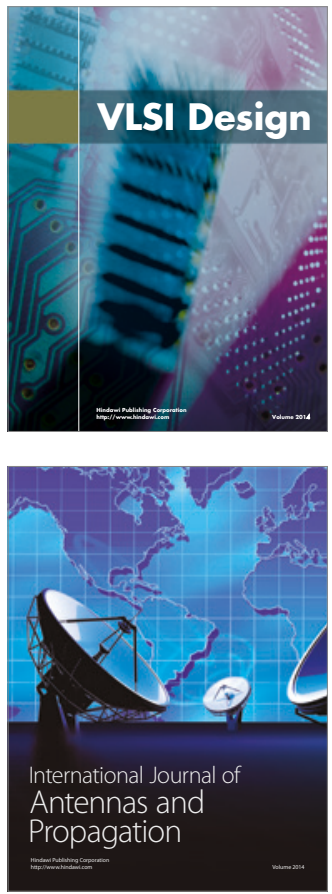

\section{Rotating}

Machinery

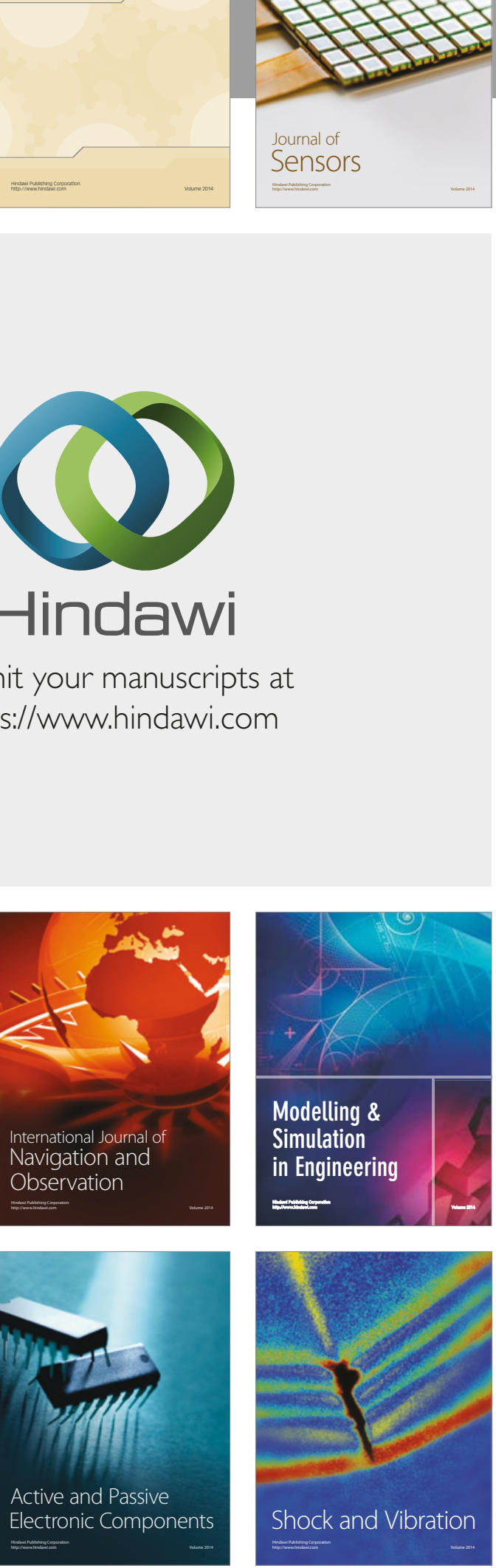
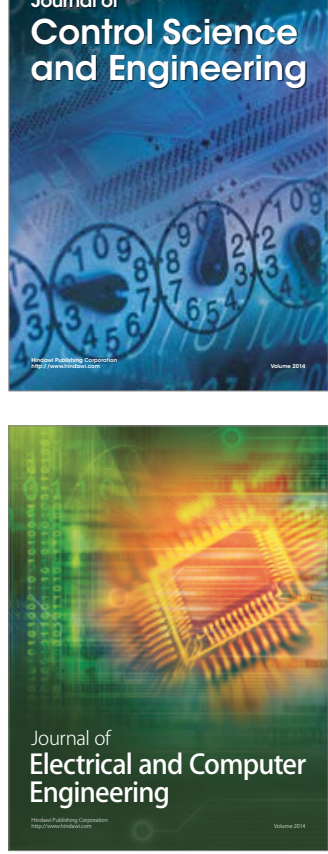

Distributed

Journal of

Control Science

and Engineering
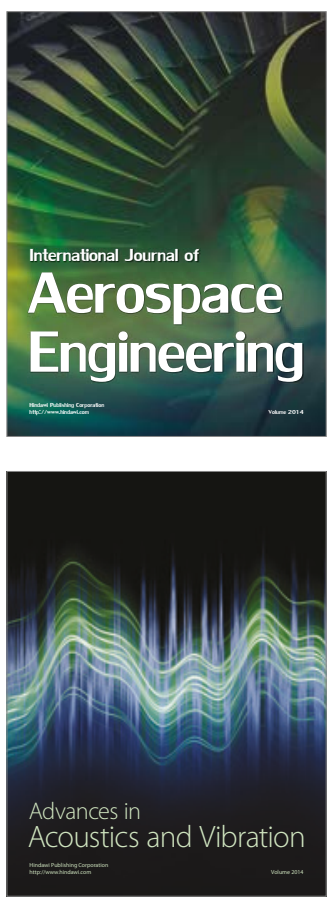

Sensor Networks 